\title{
Benign Osteogenic Neoplasm
}

National Cancer Institute

\section{Source}

National Cancer Institute. Benign Osteogenic Neoplasm. NCI Thesaurus. Code C6602.

A non-metastasizing bone-forming neoplasm. This category includes osteoma, osteoid osteoma, and osteoblastoma. 